\title{
28 Research Square \\ The epidemiology and outcome of burn injuries in Iran: A ten-year systematic review and meta-analysis
}

\section{Mohammadreza Mobayen}

Guilan University of Medical Sciences

Mohammad Ebrahim Ghaffari

Hamedan University of Medical Sciences

Fatemeh Shahriari

Guilan University of Medical Sciences

\section{Sepideh Gholamrezaie}

Guilan University of Medical Sciences

\section{Zahra Haghani Dogahe}

Guilan University of Medical Sciences

Aref Chakari-Khiavi ( $\nabla$ arefchakari@gums.ac.ir )

Guilan University of Medical Sciences

\section{Research Article}

Keywords: burn, burn epidemiology, burn outcome, systematic review, Iran

Posted Date: June 30th, 2021

DOI: https://doi.org/10.21203/rs.3.rs-505860/v1

License: (c) (i) This work is licensed under a Creative Commons Attribution 4.0 International License. Read Full License 


\section{Abstract}

Background: Burns, has always been one of the most catastrophic injuries. More than 3000000 people lose their lives every year due to burn and its results. Burn injury is the seventh major cause of disease burden of injuries in Iran. The evidence clearly shows that burns are preventable. It is necessary to update existing information and a more detailed assessment of burn aspects.

Material-Method: Web of Science, PubMed, Scopus, and Embase databases were searched for published studies from Jan. 2010 to Dec. 2020, with the MeSH term 'Burns' and keywords such as burn, burn injury, burn injuries, Iran, epidemiology, and epidemio* combined by OR; AND, for published retrospective studies that focus on the epidemiology of burns in Iran.

Results: Our systematic search identified seventeen studies containing epidemiological characteristics of burn injuries in Iran with a total number of 61662 patients. Results revealed that: The highest and lowest percentage of the burn was related to the age group of 20-29 and 0-9 years and 50-59 years; men were burned more than women. The urban residents and the person under the diploma have experienced more burns; married and single people were equally burned; burns caused by flame occurred more than the other cause of burn; male and female were burned almost equally by flame; Contact and electrical burns in men were more than women; most burn accidents occurred at home and winter; $14 / 23 \%$ patient were expired, and men were discharged more than women but the death rate was higher in women.

Conclusion: We conclude that burn injuries are common indoors, caused by flames, and in young patients with a low educational state, and the overall mortality of female burn victims is more remarkable than male burn victims. On the other hand, the mean TBSA of burn patients in Iran was 31\%, and the mean rate of mortality was $14 \%$, both of which are greater than the statistics reported by WHO. This review shows that further research is required to summarize the situation and the effectiveness of any preventive interventions.

\section{Background}

One of the most growingly recognized public health issues is injuries that considerably influencing almost every population and every region all over the globe. Burns, having always been one of the most catastrophic injuries, cause death as well as substantial economic and psychological impressions and long-term somatic sequelae. (1) Among unexpected incidents such as after road accidents and people fighting, the burn is in the fourth place. (2)

The WHO International Classification of Diseases (version 10) classifies burn injuries etiologically into categories such as exposure to smoke, fire, and flames, contact with heat and hot materials, exposure to electricity, and lighting. $(3,4)$ As stated by WHO, more than 3000000 people lose their lives every year due to burn and its results. (2) Several studies revealed the fact that some parameters, including lifestyle and the social, economic, and cultural level of society, influence the quantity and sort of burn. (5) Burn incidence and burn death rates are higher in low and middle-income countries than in wealthier countries. (6) Iran is a low-income country in the Middle East with a high rate of burn accidents and its devastating results, deaths, and disabilities. (7) Burn injury is the seventh major cause of disease burden of injuries in Iran. (8) According to epidemiological research, there are about 100,000 burn injuries every year in Iran, a country with a population of 80 million that has 10 Burn centers in 9 out of 31 provinces and 40 burn wards in hospitals. (9) Despite economic developments and also advances in the treatment of burns, it should be noted that the evidence clearly shows that burns are preventable. (10) 
Earlier studies have been conducted to focus on burns and specific groups of burn patients in Iran. now it is necessary to update existing information and a more detailed assessment of burn aspects. $(1,11)$

Considering the importance of burn prevention and the lack of a systematic study on the epidemiology of burns in Iran during the last decade, this study aimed to report epidemiological data to have a proper and cost-effective approach to problems.

\section{Material - Method}

This systematic review was conducted to describe the epidemiology and outcome of burn injuries in Iran from 2010 to 2020.

\subsection{Source of data and search Strategy}

This review conducted and reported under Preferred Reporting Items for Systematic Reviews and Meta-Analyses (PRISMA) guidelines. (12)

Web of Science, PubMed, Scopus, and Embase databases were searched for published studies from Jan. 2010 to Dec. 2020, with the MeSH term 'Burns' and keywords such as burn, burn injury, burn injuries, Iran, epidemiology and, epidemio* combined by OR; AND, for published retrospective studies that focus on the epidemiological aspects of burns in Iran. Besides, a manual search was performed.

The collected 554 potentially relevant articles were imported into the Endnote software package and checked to avoid duplication.

Moreover, the references of the retrieved articles were reviewed for further relevant studies and contributed as a source of the additional records.

\subsection{Selection criteria $\rightarrow$ Inclusion/exclusion criteria}

The selection and assessment of each study retrieved in the literature search were conducted by two investigators using the Cochrane search strategy from the scientific of Science Topics (Title, databases of Web Abstract, Keywords). (13) If any of the articles were potentially relevant, the full text of the article was reviewed.

The full manuscripts of 30 articles selected in screening were assessed for inclusion independently by both researchers using the inclusion/exclusion criteria. When there were any ambiguities, the article was handed to a third author for revision.

The main inclusion criteria were:

- Any type of retrospective study that focuses on the epidemiological aspects of burn injuries in Iran between 2010 and 2020. The records identified through the search engine contain our keywords in title, abstract or full text.

- Studies that cover burn patients of all the different variations of age and gender.

- Studies about inpatient burn victims.

The exclusion criteria were: 
- Manuscripts published before 2010.

- Review articles, case-control studies, and interventional studies (except for the introduction and discussion sections).

- Not a full manuscript (e.g., only an abstract, short communication, etc.).

- Full manuscripts not accessible.

- With epidemiology of only the deceased/forensic manuscripts.

- Articles that contain information about outpatient burn victims.

- Articles that focus on a specific age group.

- Studies about intentional or suicidal burn injuries.

- Military and war-related burns.

- Articles repeating data from other articles already included.

- Non-English language articles.

- Non-human study subjects.

\subsection{Quality assessment}

A quality assessment form was designed to extract data for a review based on a modified version of the JBI critical appraisal checklist for analytical cross-sectional studies that are essential for describing the studies' contents. In the original version of the JBI checklist, there were eight items, but two of them were not applicable in this study. (14)

Each article was given a quality score from 0 to 6 based on the details of the mentioned checklist considering the quality of reporting, external validity, and internal validity (bias and confounding). Only studies with more than 4 scores were likely to be selected in our review. 17 out of 19 studies that fulfilled the inclusion criteria also fulfilled quality assessment.

\subsection{Data extraction}

Data were extracted from the full manuscript of the 17 articles by both reviewers independently and compared to identify discrepancies using a standard data extraction spreadsheet. Data abstracted included the title, first author, date of the study, sample size, region of the study, patient demographics and burn characteristics, in-hospital events, clinical outcomes were extracted for analysis.

\subsection{Data analysis}

A meta-analysis integrates the quantitative findings from separate but similar studies and provides a numerical estimate of the overall effect of interest. (15) If the Heterogeneity test is significant, the random-effects model is used in the calculation. MedCalc uses a Freeman-Tukey transformation (16) to calculate the weighted summary Proportion under the fixed and random-effects model. (17) In this study, Cohran's $Q$ and $I^{2}$ statistics tests have been used to evaluate homogeneity. (18)

The generic inverse variance method was used to examine the quantitative data on average (mean $\pm \mathrm{SE}$ ). the calculation was performed with MedCalc software version 19.0.5 and the significance level in all tests is $5 \%$.

\section{Results}




\section{1. systematic search:}

Our systematic search identified seventeen studies containing epidemiological characteristics of burn injuries in Iran with a total number of 61662 patients. The included articles consisted of studies conducted in ten provinces of Iran $(9,19-33)$ and one study based on the national burn registry in Iran. (34) A flow diagram illustrating the literature search and study selection is shown in Fig. 1. Not all the studies have epidemiological data for all the parameters considered in this study.

\subsection{Age, gender, and demographics:}

The mean age of burn patients was $27.20 \pm 1.41$. The highest percentage of patients was in the age group of 2029 years $(24.26 \%)$, and $0-9$ years $(23.28 \%)$; meanwhile the lowest percentage was in the age group of $59-59$ years (5.27\%) (Table1). About $61.15 \%$ of patients were male, and $38.85 \%$ were female. $68.43 \%$ of patients were from urban areas, and the rest of them lived in rural areas. In the marital state, $47.03 \%$ were single, $48.58 \%$ were married, and the rest of the patients had an unknown married condition. In terms of educational state, $51.64 \%$ of patients were illiterate or didn't get a diploma, $42.12 \%$ had a diploma, $4.88 \%$ were highly educated, and the rest had an unknown educational state.

Table 1

Distribution of frequency proportion for age in total population and expired patients

\begin{tabular}{|clll|}
\hline \multicolumn{2}{|c|}{ Proportion (\%) } & expired patients & total \\
\hline age & $0-9$ & $2.83 \%$ & $23.28 \%$ \\
\cline { 2 - 4 } & $10-19$ & $22.29 \%$ & $14.36 \%$ \\
\hline $20-29$ & $29.82 \%$ & $24.26 \%$ \\
\hline $30-39$ & $17.04 \%$ & $14.65 \%$ \\
\hline $40-49$ & $7.01 \%$ & $9.72 \%$ \\
\hline $50-59$ & $7.08 \%$ & $5.27 \%$ \\
\hline$\geq 60$ & $7.65 \%$ & $6.56 \%$ \\
\hline
\end{tabular}

\subsection{Characteristics of burn injuries:}

Among the main causes of burn, flame (52.49\%) and scalds (29.17\%) had more incidence, while patients with chemical burn (2.61\%) were less than others (Table 2). In both male and female patients separately, the flame was the first, and scalds were the second common cause of burn injuries. As male patients most frequently burned with flames, the incidence of burn injuries caused by scald was higher in female patients. Male patients suffered 4.46 $(P<0.001,95 \% \mathrm{Cl}=3.76-5.29)$ times more from electrical burn injuries and $1.54(P=0.035,95 \% \mathrm{Cl}=1.03-2.31)$ times more from the burn with contact agents than female burn patients. More details about the gender distribution of the causes of the burn injuries are available in Table 3. Mean TBSA (Total body surface area) was $31.28 \pm 5.63$, and the rate of Inhalation injury was $4.56 \%$ in patients. $75.05 \%$ of burn traumas occurred at home, $14.67 \%$ of them in the workplace, and $9.88 \%$ somewhere other than home or workplace. The highest number of burns occurred in winter $(28.54 \%)$, followed by summer $(24.34 \%)$, spring $(23.60 \%)$, and autumn $(23.33 \%)$. 
Table 2

Distribution of frequency proportion for causes of burn in total population and by gender.

\begin{tabular}{|lllllll|}
\hline Proportion (\%) & \multicolumn{2}{l}{ cause } & & & & \\
\cline { 3 - 7 } & & flame & contact & scalds & electricity & chemical \\
\hline \multirow{2}{*}{ gender } & male & $51.95 \%$ & $5.94 \%$ & $33.17 \%$ & $5.19 \%$ & $3.27 \%$ \\
\cline { 2 - 7 } & female & $49.87 \%$ & $3.95 \%$ & $40.91 \%$ & $1.01 \%$ & $3.07 \%$ \\
\hline \multirow{2}{*}{ total } & & $52.49 \%$ & $3.95 \%$ & $29.17 \%$ & $5.06 \%$ & $2.61 \%$ \\
\hline
\end{tabular}

Table 3

Distribution of frequency proportion for gender in total population and by outcome or causes of burn. $\left({ }^{\star} \mathrm{Cl}\right.$ : Confidence interval)

\begin{tabular}{|c|c|c|c|c|c|c|c|c|c|}
\hline \multicolumn{2}{|c|}{ Proportion (\%) } & \multicolumn{4}{|l|}{ cause } & \multicolumn{3}{|c|}{ outcome } & \multirow[t]{2}{*}{ total } \\
\hline & & flame & contact & scalds & electricity & chemical & discharged & dead & \\
\hline \multirow[t]{2}{*}{ gender } & male & $64.24 \%$ & $71.20 \%$ & $59.58 \%$ & $86.67 \%$ & $61.96 \%$ & $63.59 \%$ & $41.62 \%$ & $61.15 \%$ \\
\hline & female & $35.76 \%$ & $28.80 \%$ & $40.42 \%$ & $13.33 \%$ & $38.04 \%$ & $36.41 \%$ & $58.38 \%$ & $38.85 \%$ \\
\hline \multicolumn{2}{|c|}{$\begin{array}{l}\text { Odds ratio }\left(\mathrm{Cl}^{*}\right. \\
95 \%)\end{array}$} & 1.079 & 1.544 & 0.751 & 4.460 & 1.043 & 2.023 & 2.023 & - \\
\hline \multicolumn{2}{|l|}{$p$. value } & 0.780 & 0.035 & 0.264 & $<0.001$ & 0.873 & $<0.001$ & $<0.001$ & - \\
\hline
\end{tabular}

\subsection{In-hospital events, and outcome:}

Mean LOS (Length of hospital stay) was $10.17 \pm 1.07$ days and $14.23 \%$ of patients died due to burn injuries. Female patients were dominant (58.38\%) among patients who died, while male patients had a higher rate (63.59\%) among discharged patients. According to the results, women died 2.02 times more than men $(P<0.001,95 \% \mathrm{Cl}=$ $1.70-2.40)$ (Table 3). The highest rate of mortality was in the age groups of 20-29 years (29.82\%) and 10-19 years $(22.29 \%)$, while the lowest percentage was in the age group of $0-9$ years $(2.83 \%)$.

\section{Discussion}

Burn injuries are a matter of public health issues worldwide, accounting for an estimated 180,000 deaths annually. (35) The purpose of this systematic review was to provide a better understanding of the burn injuries epidemiological patterns in Iran and to benefit health care providers and researchers in further studies. This review mainly focuses on statistical features containing age distribution, gender distribution, residency, marital state, educational state, TBSA, burn cause, place where the injury happened, seasonal distribution, length of hospital stay, and outcome. The findings presented in our study are similar to those in previous reviews; we extract and analyze some other remarkable data as well.

\subsection{Main findings}

This systematic review summarizes the epidemiological pattern of burn injuries in Iran as reported by seventeen studies from 2010 to 2020. 
The highest incidence of burns was in the age group of 20-29 years (the mean age was $27.20 \pm 1.41$ years old), and the second-highest incidence was in the age group of 0-9 years. These findings are in accordance with the WHO fact sheet in which both children and female adults are particularly more vulnerable to burn injuries. (35)

Adults are more likely to become burn victims as they are the main force of production lines. It is predicted that in young adults, the increased number of burn accidents in their job environments is due to insufficient experience, ignorance of the standard operating procedures at the workplace, and poor education of young workers. In other words, the reaction and coordination abilities of older adults decrease the incidence of severe burn injuries, which can explain the reduced incidence of burn injuries in older adults. The higher rate of Retirement and Unemployment in the geriatric population can be another factor justifying the lower rate of severe burn injuries.

Pediatric burn patients maintain a high percentage of patients of all age groups. (36) Young children are easily injured because of their curiosity and their explorative manners about what surrounds them. Burn injuries can cause both physical and psychological damages to children and their families. Therefore, remarkable attention ought to be paid to children, especially children under the age of 7. It is also important to limit their access to threatening items such as hot liquids or electrical outlets.

Most of our reviewed articles reporting gender distribution of burns state that males are more prone to burn injuries. In comparison with female adults, male adults work more frequently in heavy industries involving higher occupational risks of burns. According to economic changes in the past few years, women have been working outdoors and in working places that have higher risks for burn injuries as well. Despite the previous discussion, WHO mentions the higher risk of burn in females due to open fire cooking, and inherently unsafe cookstoves, which can ignite loose clothing. (35)

Residing in urban areas brings up more burn events in comparison to rural areas. Industrialization can be one of the most critical factors in cities, which can be the context of burn injuries. despite the decreased use of open flames for heating and lighting in urban areas, using electrical and chemical agents in both home and workplace for various applications increase the risk of different kinds of burn injuries. Nonetheless, in our reviewed articles, there was no enough data and evidence of the predominance of any specific cause of burn in different residency states.

There is no specific difference in the incidence rate of burn in different marital states.

The educational state shows an inverse relationship between the level of literacy and the occurrence of burn. People with higher education have better knowledge and crisis management in challenging situations. In other words, when people educate for Emergencies they can avoid precarious conditions and actions and organize the problems at the time of an accident more successfully and these educations might not have been given to people with lower education levels.

Thermal injury by flames primarily and scalds secondarily are reported to be the predominant causes of burns in most of the reviewed studies.

Thermal injury by flames is reported more in male patients rather than females, which can be associated with the occupational state of the men and more exposure to flames.

Scald is regarded as the leading cause of burn injuries in most studies, because of people's preference in drinking hot water and food, taking hot baths, eating hot pot, etc. (24)(37) it was expected to see burn injuries caused by 
scalds more in women rather than men, however with the changes in societies and the fact that women no longer work only inside the house, we did not detect a specific difference between men and women in the matter of burning with hot liquids.

Electrical burns are less common compared to the two previous kinds. In general, it causes a more severe burn injury, and it leads to a higher incidence of full-thickness burns and limb amputation, which brings great harm to burn victims and their society. (38) The electrical burn is more common in males, possibly due to their work environment.

Contact and chemical burns are less common, which can be the result of increasing literacy levels in reading the complications of abusing chemicals. Both causes are more common in males than females.

The information about the places of burn accidents is one of the most important factors that influence providing safety protocols to prevent or decrease the damage caused by burn injuries. Burns occur mainly at home and the workplace. Children and women usually burn in domestic kitchens, from upset receptacles containing hot liquids or flames from cookstove explosions. Men are most likely to burn at the workplace due to fire, scalds, chemicals, and electrical burns. (35) According to our review, burns occur at home more than at the workplace; This can suggest the necessity of training and warning parents, baby sitters, children, or any other members of the household through any national media and also providing specific instructions for the home appliance industry and delivery service providers to increase the safety grade of houses. Since there were not enough articles focused on this issue in Iran, it is suggested that future studies focus on the place of injury more than before.

Burn incidence is not comparatively different in 4 seasons. still, our data shows more occurrence in winter since people tend to use more heating equipment and in second place, summer in which people wear fewer clothes in the hot weather, as a result, they have much less protection against hot objects. Winter is the cold season in Iran. In some areas in which a regular supply of electricity is not available or where electricity is less affordable than other means, households are more likely to use kerosene devices for space heating, heating water, and making hot drinks. Household members are also expected to spend a more significant proportion of their time indoors in cold weather, which may explain the winter excess of burn injuries.

LOS is related to gender, age, anatomical site of the injury, TBSA, and other factors. Since both LOS and TBSA corresponding data in our eligible reviewed articles were not homogenous, we can just report the mean LOS of $10.17 \pm 1.07$ and mean TBSA of $31.28 \pm 5.63$. However, the actual proportion of minor and moderate burn patients might be much higher because many of the minor burns are diagnosed and treated outpatient. The outpatients also have a lower mortality rate compared to severe burn groups treated at hospitals.

Inpatient burn injuries are challenging to treat, requiring a prolonged hospital stay, costing outstanding expenses, and the higher mortality rate they carry. And as a result, improving the living standards of extensively burned victims after discharge is equally essential as decreasing their mortality rate. It is a global challenge requiring years of effort. Mortality of burns is an important indicator of the level of treatment in a region and it may be affected by many factors, such as age, gender, cause, and severity of burn injury, rescue conditions before hospitalization, complications, and state of health before the injury. This result also points out the direction of future research and the challenges standing in the way of studies of burn treatments. Our data showed that men patients survive burn injuries much greater than females, as the rate of discharge is higher in male patients. As we were not able to access the specific data of TBSA, burn degree, and anatomical site of the injury - as the severity factors of burn - 
for each gender group separately, it is hard to determine the level of rehabilitation, recovery, and lethality in two genders and explain the possible reasons of higher death rate in women.

\subsection{Implications for burn prevention}

There are some suggestions on burn prevention drawn from the epidemiological features described above: (a) Public education to warn people about the profound damages of burns and that most burn accidents are preventable. (b) A national dynamic monitoring system of burn accidents to implement the preventive methods and measures and decrease burn incidence, mortality, and disability. (c) The WHO plans for burn prevention and care (35) enclosed fires and limits for the height of open flames in domestic environments, promotion of less hazardous fuels and safer cookstoves particularly concerning stability and prevention of access by children, education regarding loose clothing while cooking, applying safety regulations to housing designs and materials, Lower temperature in hot water taps, Promotion of fire safety education and the using of smoke detectors, fire sprinklers, and fire-escape systems in homes, introduction of and compliance with industrial safety regulations, using of fire-retardant fabrics for children's sleepwear, Avoiding smoking in bed, Public Awareness about epilepsy, particularly in developing countries, Encourage further development of burn-care systems, including the training of healthcare providers in the appropriate triage and management of people with burns, and Support the development and distribution of fire-retardant aprons and using them while cooking around an open flame or kerosene stove.

\subsection{Limitations of this study}

1. as a result of lack of sufficient information, this study did not provide a complete review of crude burn incidence; Not all articles contained comprehensive and detailed data on all of the elements we reviewed, and there were also insufficient data on outpatients.

2. This review is not able to show the epidemiological pattern and the differences in various aspects entirely because the method of research and the distribution of age, agents, the severity of burns (burn degree), and anatomical site of the injury vary in various selected studies. In other words, there was considerable heterogeneity in the way studies reported their findings which limited some of the comparisons made in our research. Improved data collection systems of nationwide and population-based surveys will help to solve the problem.

Recommendations for research and practice Standardization of burn epidemiological studies would help improve the quality of such studies. Reporting studies in line with the STROBE (Strengthening the Reporting of Observational Studies in Epidemiology) guidelines would address many of the deficiencies we found in the quality of the reporting of studies and journals publishing epidemiological studies should require following such guidelines. Also, to use these guidelines, there is some specific information about burns that would be helpful if included in epidemiological studies of burn injuries. this information consists of reporting age groups in line with the WHO injury reports, reporting the distribution of the total body surface area of burn injury more extensively than merely providing the mean or median (e.g., at least report quartiles or quintiles), and reporting mortality considering the diverse spectrum of TBSA.

Further researches are required to systematically review the effectiveness of interventions and preventions of common thermal injuries in Iran. Following this, burn prevention strategies need to be revised, and prevention programs need to be developed, especially addressing specific burn injury mechanisms in high-risk settings and high-risk groups. 
3. This research is not providing Data on Pre-hospital emergency management of burn patients as a result of the lack of sufficient information; Therefore, future researches need to pay more attention to this part to determine gaps in the healthcare systems. More prevention-oriented studies on burn incidence, and studies with attention to socioeconomic factors such as occupation and income, comorbidities, past medical history, length of hospital stay (LOS), length of ICU stay, wound infection, the use of blood products, treatment strategies, consequences of burns, quality of the survivor's life and rehabilitation, should be conducted. Besides, it would be better to have a standard classification of age, etiology, severity, anatomical site, etc., in future studies.

4. In some of the reviewed articles, inhalation injury is considered as a separate cause of the burn along with other causes of burn injuries and not as a specific type of injury that happens mostly when people burn with the flames. Inhalation injury alone is one of the factors increasing the length of hospital stay, particularly in people with other concomitant burn injuries. Therefore, it is necessary to consider and report it as a separate type of injury and not a discrete reason for injury in burn epidemiological studies.

5. There were also several parameters that we were willing to evaluate, like treatment strategies, but since the lack of data, the evaluation was not possible

6. As with any systematic review, the data obtained from studies is in part dependent on the quality of the reporting and in part on the quality of the researches.

\subsection{Strengths of the study}

1. We used a standard systematic review methodology, including literature search, the selection of studies for inclusion, data extraction, and quality assessment of a sample of the studies by two independent reviewers, and get help from a Statistics specialist to reach a more accurate quantity assessment.

2. We restricted our review to the published articles indexed in Scopus, Embase, PubMed, and Web of Science.

3. Unpublished studies or those published in journals not indexed in the databases above were not considered in our review due to avoid possible bias and confounding.

\section{Conclusion}

In this study, we conclude that burn injuries are commonly caused by flames, indoors, and in young patients with a low educational state. the overall mortality of female burn victims is more remarkable than male burn victims. the mean TBSA of burn patients in Iran was $31 \%$, and the mean rate of mortality was $14 \%$, both of which are greater than the statistics reported by WHO. Burn injuries are one of the leading causes of trauma in developing countries, including Iran. The lack of an organized registration program can act as an obstacle in the way of knowing the pattern of burn injuries among different population groups and developing prevention programs. Despite our efforts on collecting epidemiological information on burn injuries from all over Iran, we found out that several burn centers in Iran have not yet published information about the epidemiology of burn injuries at least in the last decade. This fact shows the necessity of similar studies in certain regions in Iran. Further researches are required to summarize the situation and the effectiveness of any preventive interventions undertaken in order to provide evidence for developing and implementation burn prevention programs. Besides, increased public awareness and education are necessary to decrease the incidence of these potentially catastrophic injuries.

\section{Abbreviations}


TBSA: Total body surface area, LOS: Length of hospital stay

\section{Declarations}

7.1 Ethics approval and consent to participate

Not applicable

7.2 Consent for publication

Not applicable

7.3 Availability of data and materials

The datasets used and analyzed during the current study are available from the corresponding author on reasonable request.

7.4 Competing interests

No competing interests to declare.

7.5 Funding

Self-funding.

7.6 Authors' contributions

All authors were involved in designing this study. AC-K, FS, and MM designed the search protocol. AC-K, FS, and SG performed the screening and selection of articles and collected the data. ZHD reviewed the quality of all included articles. MEG performed the data analyses, and it was checked by AC-K and MM. All authors were involved in structuring and writing the manuscript. All authors read and approved the final manuscript.

7.7 Acknowledgements

We wish to thank Burn and Regenerative Medicine Research Center, Guilan University of Medical Sciences, for their kind assistance in performing this study.

\section{References}

1. Sadeghi-Bazargani H, Mohammadi R. Epidemiology of burns in Iran during the last decade (2000-2010): Review of literature and methodological considerations. Burns. 2012.

2. Kumar S, Ali W, Verma AK, Pandey A, Rathore S. Epidemiology and mortality of burns in the Lucknow Region, India - A 5 year study. Burns. 2013;

3. World Health Organisation W. Burn-out an "occupational phenomenon": International Classification of Diseases. Int Classif Dis. 2019; 
4. Othman N, Kendrick D. Epidemiology of burn injuries in the East Mediterranean Region: A systematic review. BMC Public Health. 2010;

5. Albertyn R, Bickler SW, Rode H. Paediatric burn injuries in Sub Saharan Africa-an overview. Burns. 2006;

6. Al-Shehri M. The pattern of paediatric burn injuries in Southwestern, Saudi Arabia. West Afr J Med. 2004;

7. Matin BK, Rezaei S, Karyani AK. Factors associated with length of stay and hospital charges among pediatric burn injury in Kermanshah, west of Iran. Int J Pediatr. 2015;

8. Emami SA, Motevalian SA, Momeni M, Karimi H. The epidemiology of geriatric burns in Iran: A national burn registry-based study. Burns. 2016;

9. Alavi CE, Salehi SH, Tolouei M, Paydary K, Samidoust P, Mobayen M. Epidemiology of burn injuries at a newly established burn care center in Rasht. Trauma Mon. 2012;

10. Keshavarz M, Javanmardi F, Mohammdi AA. A Decade Epidemiological Study of Pediatric Burns in South West of Iran. World J Plast Surg. 2020;

11. Hashemi SS, Sharhani A, Lotfi B, Ahmadi-Juibari T, Shaahmadi Z, Aghaei A. A systematic review on the epidemiology of pediatric burn in Iran. J Burn Care Res. 2017;

12. Moher D, Liberati A, Tetzlaff J, Altman DG, Altman D, Antes G, et al. Preferred reporting items for systematic reviews and meta-analyses: The PRISMA statement. PLoS Medicine. 2009.

13. Higgins JPT, Thomas J, Chandler J, Cumpston M, Li T, Page MJ, Welch VA (editors). Cochrane Handbook for Systematic Reviews of Interventions. 2nd Edition. Chichester (UK): John Wiley \& Sons, 2019.

14. JBI Reviewer's Manual - JBI Manual for Evidence Synthesis - JBI GLOBAL WIKI [Internet]. [cited 2021 Jan 20]. Available from: https://wiki.jbi.global/display/MANUAL/JBI+Reviewer\%27s+Manual

15. Petrie A, Bulman JS, Osborn JF. Further statistics in dentistry Part 8: Systematic reviews and meta-analyses [Internet]. Vol. 194, British Dental Journal. Nature Publishing Group; 2003 [cited 2021 Jan 21]. p. 73-8. Available from: https://www.nature.com/articles/4809877

16. Freeman MF, Tukey JW. Transformations Related to the Angular and the Square Root. Ann Math Stat. 1950;

17. DerSimonian R, Laird N. Meta-analysis in clinical trials. Control Clin Trials. 1986;

18. Higgins JPT, Thompson SG, Deeks JJ, Altman DG. Measuring inconsistency in meta-analyses. British Medical Journal. 2003.

19. Matin BK, Najafi F, Mahboubi M, Ahmadi-Jouybari T, Eskandari S, Moradinazar S. A study on burn Epidemiologic Situation in Kermanshah, Iran in 2015. Res J Appl Sci. 2016;

20. Hosseini SN, Rashtchi V, Kamali K, Moghimi MH. Epidemiology and outcome of 2,590 burned patients in Northwest Iran. Ann Burns Fire Disasters. 2017; 
21. Ekrami A, Hemadi A, Kalantar E, Latifi M, Kayedani A. Epidemiology of hospitalized burn patients during 5 years in Khuzestan province, Iran. Iran J Clin Infect Dis. 2010;

22. Mohammadi-Barzelighi H, Alaghehbandan R, Motevallian A, Alinejad F, Soleimanzadeh-Moghadam S, Sattari $M$, et al. Epidemiology of severe burn injuries in a Tertiary Burn Centre in Tehran, Iran. Ann Burns Fire Disasters. 2011;

23. Khazaei S, Shirani F, Afshari M, Jenabi E, Hamzei Z, Torabi M, et al. Etiology and outcome of burns in Hamadan, Iran: A registry-based study. Arch Trauma Res. 2019;

24. Sadeghi Bazargani H, Arshi S, Ekman R, Mohammadi R. Prevention-oriented epidemiology of burns in Ardabil Provincial Burn Centre, Iran. Burns. 2011;

25. Karimi H, Momeni M, Motevalian A, Bahar MA, Boddouhi N, Alinejad F. The burn registry program in Iran First report. Ann Burns Fire Disasters. 2014;

26. Goodarzi M, Reisi-Dehkordi N, Daryabeigi R, Zargham-Boroujeni A. An epidemiologic study of burns: Standards of care and patients' outcomes. Iran J Nurs Midwifery Res. 2014;

27. Bahar MA, Pakyari M, Bahar R. Burns in Tehran: Demographic, etiological, and clinical trends. Asian Biomed. 2014;

28. Shahabi Shahmiri S, Kolahdouzan M, Omrani A, Khazaei M, Salehi H, Motavalian A, et al. Determinants of Mortality and the Lethal Area 50 Index (LA50) in Burn Patients Admitted to a Large Burn Center; A Single Center Experience. Bull Emerg trauma. 2017;

29. Karami MB, Matin KR, Joybari AT, Ghahvehei N, Haghi M, Ahmadi M, et al. Epidemiological data, outcome, and costs of burn patients in kermanshah. Ann Burns Fire Disasters. 2012;

30. Ahmadi A, Ruchi AB, Soltani N, Iranagh JA, Motalebi SA. Epidemiological study of 1938 burn patients from 2014-2016 in Qazvin, Iran. Trauma Mon. 2020;

31. Shirkhoda M, Kaviani Far K, Narouie B, Shikhzadeh A, Ghasemi Rad M, Hanfi Bojd H. Epidemiology and evaluation of 1073 burn patients in the southeast of Iran. Shiraz E Med J. 2011;

32. Danesh HA, Javanbakht S, Nourallahzadeh M, Bakhshani NM, Danesh S, Nourallahzadeh F, et al. Epidemiology and mortality of burn injuries in Eastern Iran since 2009: An analysis of 2115 cases. Int J High Risk Behav Addict. 2019;

33. Alipour J, Mehdipour Y, Karimi A. Epidemiology and outcome analysis of 3030 burn patients with an icd-10 approach. Ann Burns Fire Disasters. 2020;

34. Rezaee R, Alimohamadzadeh K, Hossini S-M. Epiemiologic Features and Hospitalization Cost of Burn Injuries in Iran Based on National Burn Registry; a Cross-sectional Study. Arch Acad Emerg Med. 2019;

35. Burns [Internet]. [cited 2021 Jan 20]. Available from: https://www.who.int/news-room/factsheets/detail/burns 
36. L. K-Y, X. Z-F, Z. L-M, J. Y-T, T. T, W. W, et al. Epidemiology of pediatric burns requiring hospitalization in China: A literature review of retrospective studies. Pediatrics. 2008;

37. Den Hertog PC, Blankendaal FACM, Ten Hag SM. Burn injuries in The Netherlands. Accid Anal Prev. 2000;

38. Depamphilis MA, Cauley RP, Sadeq F, Lydon M, Sheridan RL, Driscoll DN, et al. Surgical management and epidemiological trends of pediatric electrical burns. Burns. 2020;

\section{Figures}
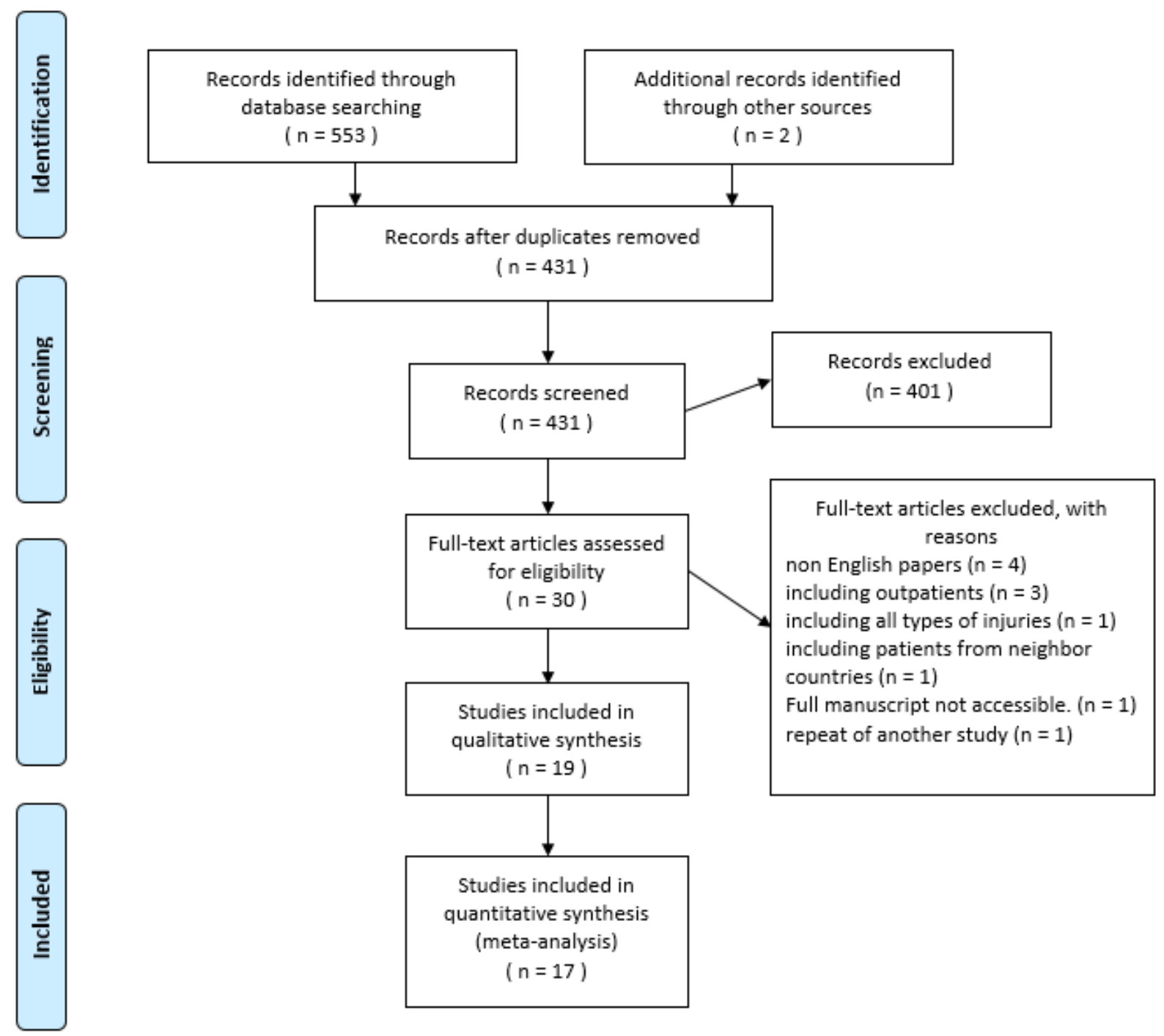

\section{Figure 1}

Flow chart of study selection for inclusion in the meta-analysis. 